\title{
Visual Estimation of Force Applied During Simulated Deliveries Complicated by Shoulder Dystocia
}

\author{
Margaret Walters, LT, MC, USN ${ }^{1} \quad$ Allison Eubanks, LT, MC, USN ${ }^{1}$ Elizabeth Weissbrod, MA, CMI \\ John Fischer, Col, MC, USAF ${ }^{1}$ Barton Staat, Lt Col, MC, USAF ${ }^{1}$ Shad Deering, COL, USA, MC ${ }^{1}$ \\ ${ }^{1}$ Department of Obstetrics and Gynecology, Walter Reed National \\ Military Medical Center, Bethesda, Maryland \\ 2 The Henry M. Jackson Foundation for the Advancement of Military \\ Medicine, Inc.; Department of Obstetrics and Gynecology, \\ Uniformed Services University of the Health Sciences, Bethesda, \\ Maryland \\ Am J Perinatol Rep 2018;8:e206-e211. \\ Address for correspondence Allison Eubanks, MD, Department of \\ Obstetrics and Gynecology, Walter Reed National Military Medical \\ Center, Bethesda, MD (e-mail: allison.a.eubanks.mil@mail.mil).
}

\begin{abstract}
Keywords

- shoulder dystocia

- training

- traction

- fetal injury

- simulation

- legal

Background Shoulder dystocia occurs when the fetal head delivers, but the shoulder is lodged behind the pubic symphysis. Training for these emergency deliveries is not optimized, and litigation can occur around a shoulder dystocia delivery.

Objective Evaluate the ability of an outside observer to visually estimate the amount of traction applied to the fetal head during simulated deliveries complicated by shoulder dystocia.

Study Design Simulated deliveries with an objective measurement of traction were randomly organized for estimation of traction applied. Videos show providers applying a "normal" $(75 \mathrm{~N})$ and "excessive" $(150 \mathrm{~N})$ amount of force in both a "calm" and "stressed" delivery.

Results Fifty participants rated the amount of force applied. Observers estimated traction, on a scale from 1 to 5 , higher in the $150-\mathrm{N}$ deliveries as compared with $75-\mathrm{N}$ deliveries ("calm" environment: 3.1 vs. $2.8, p<0.001$; and "stressed" environment: 3.2 vs. $2.8, p<0.001$ ). Only $15 \%$ of observers rated force "above average" or "excessive" in a "calm" environment, as opposed to $30 \%$ of observers in the "stressed" environment.

Conclusion Observers are not able to determine when "excessive force" is used and are twice as likely to overestimate the force applied to a fetal head when an average amount of force is used and the delivery environment is stressful.

Precis Observers are unable to determine when excessive traction is applied to the fetal head during simulated deliveries complicated by shoulder dystocia.
\end{abstract}

Shoulder dystocia is a true obstetric emergency that occurs when the fetal head delivers, but the anterior shoulder is lodged behind the pubic symphysis. Specific maneuvers to reduce the dystocia, to include rotation of the fetal shoulders or delivery of the posterior arm, as Grimm et al showed, can reduce the delivery force and the amount of stretch placed on the brachial plexus. ${ }^{1}$ Despite the use of these maneuvers, it is often necessary to apply some traction on the fetal head to

received

March 2, 2018

accepted after revision

August 21, 2018
DOI https://doi.org/

10.1055/s-0038-1673377. ISSN 2157-6998. assist with delivery. There is discussion in the literature pertaining to how much traction can or should be applied, without resulting in injury to the fetus. ${ }^{1-5}$

The amount of traction applied to the fetal head can be measured during simulated deliveries or with use of special equipment during real deliveries. Data have suggested that there is a threshold of force that can result in a brachial plexus injury. Allen et al measured the amount of force used in delivery
Copyright $\odot 2018$ by Thieme Medical Publishers, Inc., 333 Seventh Avenue, New York, NY 10001, USA. Tel: +1(212) 584-4662.
License terms

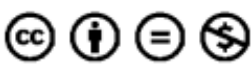


by means of a sensor attached to the provider's delivery hand and reported a single case where a force of $99.89 \mathrm{~N}$ resulted in a transient brachial plexus injury. ${ }^{2}$ Using a simulator equipped with a force monitor, Deering et al found that the mean force applied after initial maneuvers for a simulated shoulder dystocia was $67 \mathrm{~N}^{4}$ Literature also suggests that impulse or intermittent force applied may be more likely to cause nerve injuries; however, slow, steady maximal force is not well studied. Further, the question remains whether individuals observing deliveries are able to estimate the amount of traction applied to the fetal head without the use of specialized equipment. In the court room, "excessive force" based on an observer's estimate is often cited as the reason for brachial plexus injuries or clavicular fractures. ${ }^{6}$ However, there is growing literature showing that injury can occur, even when no human force is used to deliver the head. ${ }^{3,5,7,8}$ Additionally, there are no studies to support an observer's ability to determine the amount of traction applied, and if it was excessive. This is important because a reliable visual determination of excessive force would offer an opportunity for correction. To answer this question, we designed this study to investigate the ability of an observer to visually estimate the amount of traction applied to the fetal head during simulated deliveries complicated by shoulder dystocia. Secondarily, the study evaluated the impact of the delivery environment on the perceived amount of traction being applied.

\section{Materials and Methods}

The protocol was approved by the hospital institutional review board. Four providers from Walter Reed National Military Medical Center (one obstetrician/gynecologist resident and three obstetrician/gynecologist staff) volunteered to play the role(s) of the delivering provider in the videos. A PROMPT childbirth simulator was used, which measures the force applied to the fetal head during delivery. The mechanism of this simulator is described in a previous publication. ${ }^{9}$ The providers being filmed were briefed on the purpose of the study and were each given a standardized script, specifically describing their role and conduct for each of the different scenarios. During each, approximately 60-second scenario, the providers begin with a normal delivery that quickly develops into a dystocia. All videos start calm with just the nurse, patient behind the simulator, and physician, but as the dystocia progressed, the "stressed" environment videos become chaotic with lack of communication and poor execution of maneuvers. In all videos, McRobert's maneuver, suprapubic pressure, and rotational maneuvers, in combination with gentle downward traction on the fetal head, were used to relieve the dystocia and, ultimately, deliver the infant. We chose to use $75 \mathrm{~N}$ as normal or "average" force, based on the previous study performed by Deering et al, which showed $47 \mathrm{~N}$ was the average force for a normal delivery and $67 \mathrm{~N}$ was the average force for a delivery complicated by shoulder dystocia. ${ }^{4}$ To easily identify the values on the software for the simulation providers, we chose $75 \mathrm{~N}$. We then chose $150 \mathrm{~N}$ to represent "excessive" force, which is $50 \%$ higher than what Allen et al reported as being associated with a case of brachial plexus injury and twice as much as the 75-N level. The providers in the videos were able to watch a monitor off camera to ensure they were applying the specified amount of force during their scenarios.

A total of 16 videos were created, each depicting a delivery complicated by a shoulder dystocia. Each of the four providers filmed four different scenarios. The videos differed by the amount of traction being applied (75 vs. $150 \mathrm{~N}$ ) and the environment (calm vs. stressed). A "calm" environment was characterized by good communication between team members and the patient, voiced in a composed manner, and timely interventions. In contrast, a "stressed" environment was characterized by poor communication, frantic movements, and raised voices, though all of the same maneuvers were still performed. In each scenario, the baby was delivered in the occiput anterior (OA) position and restituted to right occiput transverse (ROT). The camera was positioned to the delivering provider's right side, providing a clear vantage point for the delivery and all maneuvers. In all videos, the provider requested McRobert's with suprapubic pressure from the nurse and delivery did not occur until after rotational maneuvers were applied.

Providers at our institution, to include labor and delivery nurses, midwives, interns, residents, and staff, were recruited to participate in the study at morning reports and during academics. All above providers were given the opportunity to participate, but due to schedules, off-site rotations, or other conflicts, not all providers could participate. Therefore, subjects were included if they could watch the videos during the announced times or if they arranged for other time. Subjects were included if they were older than 21 years and excluded if they were pregnant, had not seen a delivery involving a shoulder dystocia in the past, or declined to participate. After informed consent was obtained, a standardized evaluation form was provided, where the participants were instructed to complete a demographics section, to include gender, job position, area of practice, number of deliveries seen, and number of shoulder dystocia deliveries seen. Participants viewed the 16 prerecorded videos, which were placed in random order using a random number generator. These subjects were blinded to the fact that there were only two different forces used in the videos and different environments. The participants utilized a 5-point Likert scale, with provided anchors as guides, to estimate the amount of force applied during the delivery, with the scale anchors being: minimal (1), less than average (2), average (3), above average (4), and excessive (5). The Likert scale was used and weighted to examine the subjective nature of evaluating force.

Responses were recorded and the data analyzed with both chi-squared tests and a paired $t$-test as appropriate. A $p$-value of $\leq 0.05$ was considered significant.

\section{Results}

We approached a total of 55 providers to participate, 50 of whom were eligible and fully completed the evaluation forms, including 23 staff, 8 residents, 2 interns, 15 nurses, and 2 midwives. Five of those approached had not witnessed a shoulder dystocia delivery and were excluded. No participants 
Table 1 Demographics

\begin{tabular}{|c|c|c|c|c|c|c|}
\hline & Intern & Resident & Attending & Midwife & Nurse & Total \\
\hline Number of participants & 2 & 8 & 23 & 2 & 15 & 50 \\
\hline Male & 0 & 1 & 13 & 0 & 0 & 14 \\
\hline Female & 2 & 7 & 10 & 2 & 15 & 36 \\
\hline 21-50 deliveries & 1 & 0 & 0 & 0 & 3 & 4 \\
\hline $50+$ deliveries & 1 & 8 & 23 & 2 & 12 & 46 \\
\hline 1-4 shoulder dystocia (SD) & 2 & 4 & 1 & 0 & 4 & 11 \\
\hline 5-9 SD & 0 & 2 & 7 & 1 & 4 & 14 \\
\hline $10+S D$ & 0 & 2 & 15 & 1 & 7 & 25 \\
\hline
\end{tabular}

declined to participate. Forty-six participants had seen $>50$ deliveries and 24 participants had seen $>10$ deliveries complicated by shoulder dystocia. Demographics of participants can be seen in -Table 1 .

-Figs. 1 and 2 illustrate the means with the standard deviations of the responses obtained when assessing each of the four scenarios (calm environment, $75 \mathrm{~N}$; stressed environment, $75 \mathrm{~N}$; calm environment, $150 \mathrm{~N}$; and stressed environment, $150 \mathrm{~N}$ ). When comparing the mean responses from the calm $75-\mathrm{N}$ cohort and the calm $150-\mathrm{N}$ cohort, there is a statistically significant difference. However, the mean values

\section{Mean Force 75N \& 150N Calm (w/Standard Deviation)}

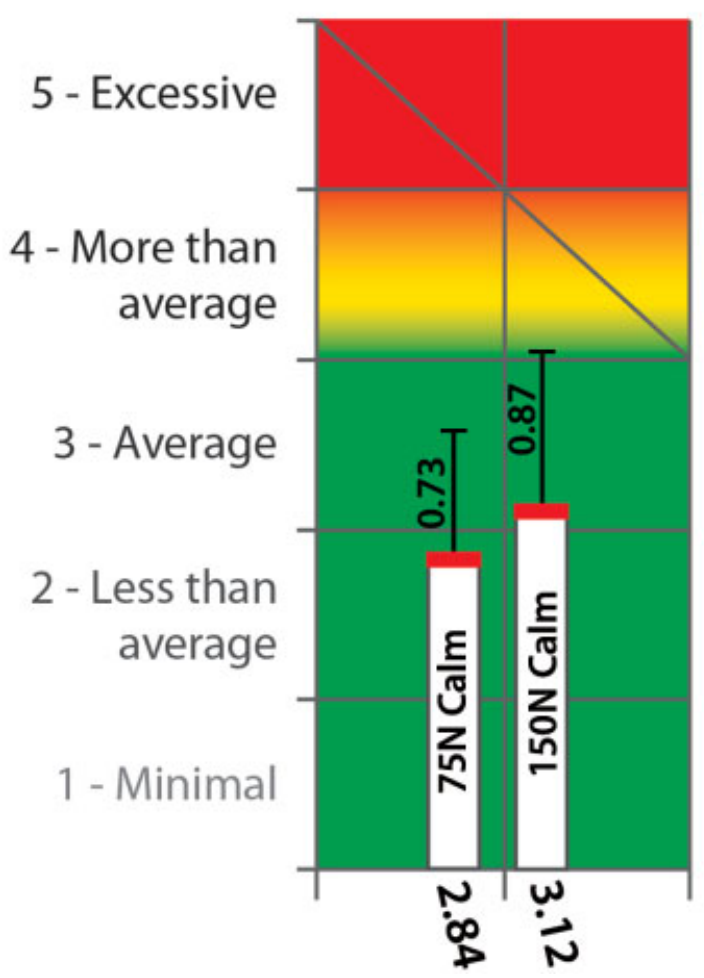

Fig. 1 Mean force 75 and $150 \mathrm{~N}$ in calm environment (with standard deviation). for these two groups were $2.8 \pm 0.73$ and $3.1 \pm 0.94$, respectively, meaning that the majority of viewers still thought the applied force in the simulated delivery was within the range of average force. The same difference was seen in the stressed 75$\mathrm{N}$ versus stressed 150- $\mathrm{N}$ cohorts, with mean values of $2.8 \pm 1.0$ and $3.2 \pm 0.87$, respectively. Again, while there is a difference, the mean values still rate the amount of applied force as "average," despite the difference in actual force applied.

With regard to effects of environment on the perception of traction, 15\% (30/200) of participants stated that "above average/excessive" force was used in the calm 75-N cohort, while 30\% (60/200) of participants stated that "above average/excessive" force was used in the stressed 75-N cohort,

\section{Mean Force 75N \& 150N Stressed (w/Standard Deviation)}

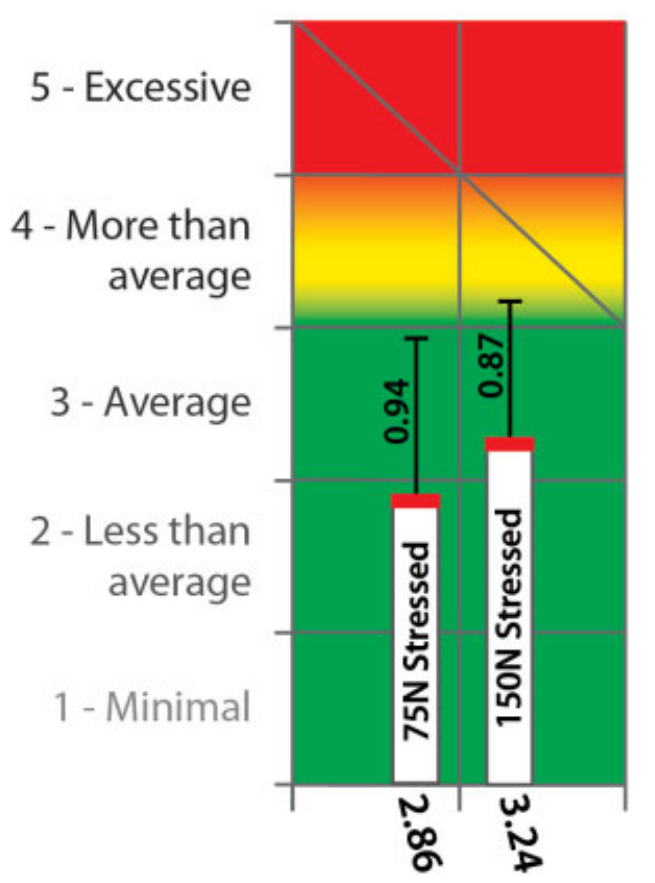

Fig. 2 Mean force 75 and $150 \mathrm{~N}$ in stressed environment (with standard deviation). 


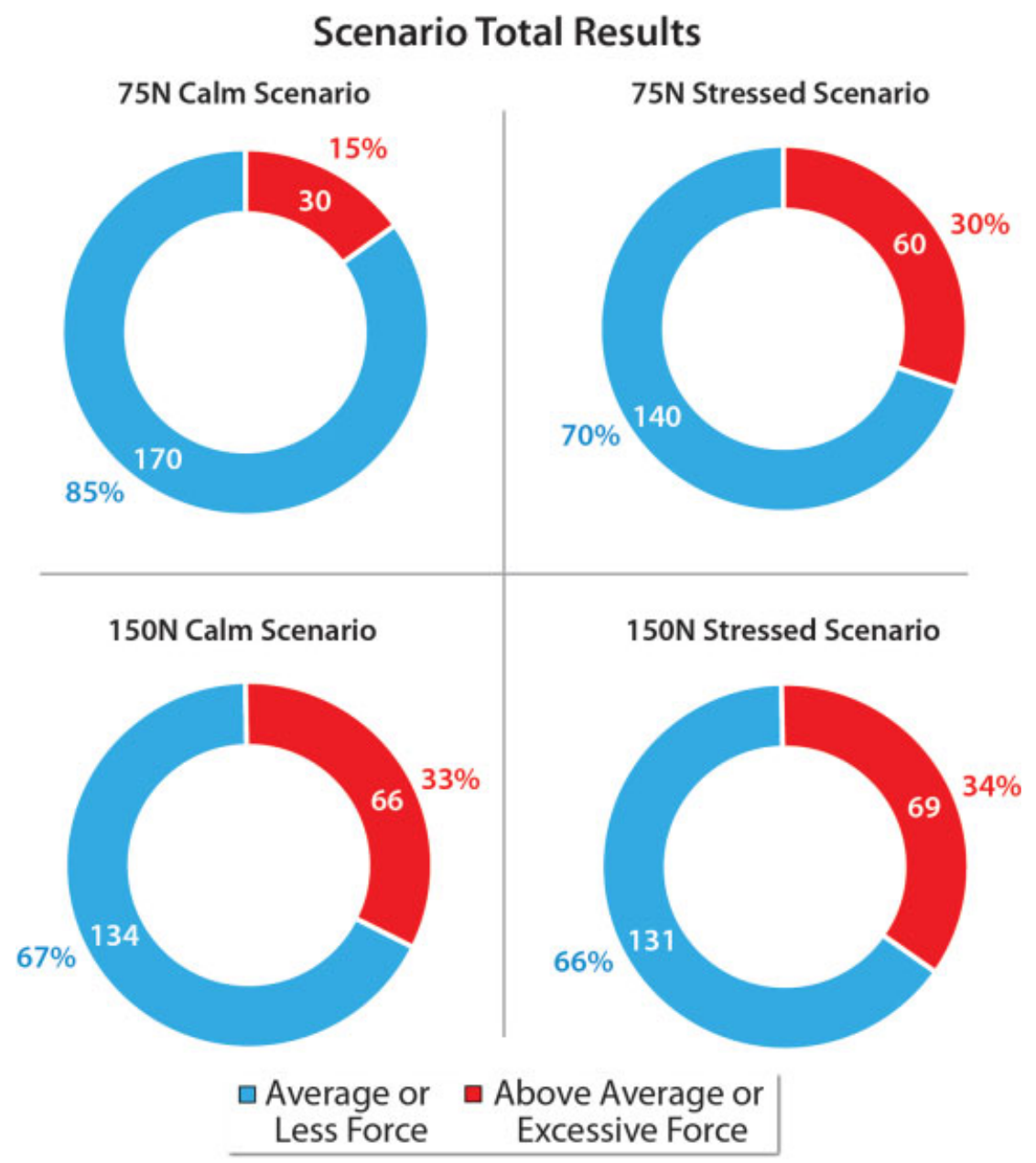

Fig. 3 Estimation of traction in all scenarios.

with a $p$-value of $<0.001$, as seen in - Fig. 3 . This difference was not observed in the $150-\mathrm{N}$ cohorts. Also shown in -Fig. 3, 33\% (66/200) of participants stated that "above average/excessive" force was used in the calm 150-N cohort and 34.5\% (69/200) of participants stated "above average/ excessive" force was used in the stressed $150-\mathrm{N}$ cohort, resulting in a $p$-value of 0.75 .

\section{Discussion}

Our study demonstrates that providers were only able to identify when excessive force was used about one-third of the time, and that when a normal amount of force was applied but the environment was "stressful," they were significantly more likely to overestimate the force applied. Although there was a statistically significant difference in the mean values of the calm 75-N cohort (2.8) and the calm 150-N cohort (3.1), both values suggest that observers thought an "average" amount of force was being applied. To reinforce that observers are unable to accurately assess when excessive force is being used, only around $30 \%$ of participants identified "above average" or "excessive" traction when viewing the $150-\mathrm{N}$ videos.

We also found that the delivery environment played a significant role in the perception of how much traction was applied. Only $15 \%$ of observers rated the amount of traction as "above average" or "excessive" in the 75-N scenarios with a calm environment, while $30 \%$ of participants indicated "above average/excessive" force in the 75-N stressed environment cohort. This suggests that despite the same amount of force being applied, viewers perceive the use of more force being applied when the environment is stressed, which we simulated by poor communication, frantic movements, and raised voices in the videos. This indicates that the chaos of the environment may cause the observer to feel more panic and desperation from the delivering provider, with the perception that the provider is applying more traction.

The evaluation for differences in ratings based on the number of deliveries or number of shoulder dystocias seen were not significantly different as shown in - Table $\mathbf{2}$, which stratifies the databased on experience.

The observers in this study had all witnessed deliveries complicated by shoulder dystocia. Even those who only witnessed one to four dystocias were included, without any experience of actually delivering a baby through a dystocia, as we feel this group may still be called on for their accounts of the events should a delivery complicated by shoulder dystocia end with an adverse outcome. We specifically chose a group of 
Table 2 Pearson's chi-squared tests based on experience

\begin{tabular}{|l|l|l|}
\hline Clinical scenario & $\begin{array}{l}\text { Number of } \\
\text { deliveries } \\
\text { seen }\end{array}$ & $\begin{array}{l}\text { Number of } \\
\text { shoulder } \\
\text { dystocias seen }\end{array}$ \\
\hline $\begin{array}{l}\text { 75-N delivery, } \\
\text { calm environment }\end{array}$ & 0.745 & 0.167 \\
\hline $\begin{array}{l}\text { 75-N delivery, } \\
\text { stressed environment }\end{array}$ & 0.486 & 0.060 \\
\hline $\begin{array}{l}\text { 150-N delivery, } \\
\text { calm environment }\end{array}$ & 0.872 & 0.593 \\
\hline $\begin{array}{l}\text { 150-N delivery, } \\
\text { stressed environment }\end{array}$ & 0.823 & 0.492 \\
\hline
\end{tabular}

providers with experience as we felt they would be the best suited to accurately estimate the amount of traction applied, given their experience. However, the data we obtained suggest otherwise. It is reasonable to assume that inexperienced observers in the room, to include the patient or the patient's family, are also unable to estimate the amount of traction. Growing literature argues that fetal injury can be due to other factors, such as prolonged labor or fetal size, as injury is seen at the time of routine deliveries and cesarean section. $3,5,7,8$ Therefore, the legal argument that "excessive force" is the cause of fetal injury seems inadequate, given that (1) witnesses are unable to tell when excessive force is being applied and (2) injury can occur without any human-applied traction on the fetal head. Further studies, to include inexperienced observers, including nonmedical personnel and even patients, would help to solidify this conclusion.

Given the inability of observers to accurately assess the amount of traction being applied during a delivery, we feel that our findings highlight the importance of training delivering providers using a birthing simulator to objectively measure the amount of force applied to the fetal head. This also falls in line with evidence in the literature to suggest that training providers with simulation can improve performance during actual deliveries, as well as decrease the incidence of fetal injury. ${ }^{10,11}$ Hopefully, allowing delivering providers to perform simulated deliveries with accurate feedback on the amount of traction applied will help them realize when they may be applying excessive traction. ${ }^{11,12}$ Additionally, the study supports the importance of remaining calm during obstetrical emergencies. Through simulation of deliveries complicated by shoulder dystocia, the delivering provider will feel more prepared to handle such situations as they arise in actual deliveries and to maintain a calm and controlled environment.

We appreciate that there are limitations to our study, namely that the videos being viewed are simulations and cannot completely mimic actual deliveries and therefore the actual traction being applied. Also, the values chosen to represent "average" and "excessive" force may not accurately represent the force in actual deliveries that represent a safe amount of traction or excessive traction. However, with the literature that does exist quantifying force used during delivery, we feel the values we assigned to "average" and "excessive" force are based on the best objective evidence currently available. Additionally, we realize that not being present for the delivery and only viewing it on video may affect the observer's perception of the amount of force applied. Finally, while there is not a previous validation study of the Likert scale used, the anchors chosen were an attempt to allow the responder to indicate the presence of excessive force and this was further strengthened by the analysis. The PROMPT simulator has been discussed in several previous publications and its use to measure force applied during simulated shoulder dystocias is well represented in the literature. ${ }^{9}$

Our study concludes that experienced observers are unable to reliably appreciate when excessive force is being applied during a simulated shoulder dystocia, and we feel that this should emphasize the need for training for management of a delivery complicated by a shoulder dystocia. In addition, a stressed environment may also contribute to an observer perceiving the amount of traction as being excessive when normal force is used, and again, we would suggest that more training may help to address this.

\section{Disclaimer}

The views expressed in this article are those of the author (s) and do not necessarily reflect the official policy or position of the Department of the Navy Department of the Army, Department of the Air Force, Department of Defense, or the United States Government.

Several of the authors are military service members. This work was prepared as part of their official duties. Title 17 U.S.C. 105 provides that "Copyright protection under this title is not available for any work of the United States Government." Title 17 U.S.C. 101 defines a United States Government work as a work prepared by a military service member or employee of the United States Government as part of that person's official duties.

\section{Meeting Presentations}

This article was presented at ACOG Armed Forces District, October 2015, Norfolk, VA.

\section{Conflict of Interest}

None.

\section{References}

1 Grimm MJ, Costello RE, Gonik B. Effect of clinician-applied maneuvers on brachial plexus stretch during a shoulder dystocia event: investigation using a computer simulation model. Am J Obstet Gynecol 2010;203(04):339.e1-339.e5

2 Allen R, Sorab J, Gonik B. Risk factors for shoulder dystocia: an engineering study of clinician-applied forces. Obstet Gynecol 1991;77(03):352-355

3 Allen RH, Gurewitsch ED. Temporary Erb-Duchenne palsy without shoulder dystocia or traction to the fetal head. Obstet Gynecol 2005;105(5, Pt 2):1210-1212

4 Deering SH, Weeks L, Benedetti T. Evaluation of force applied during deliveries complicated by shoulder dystocia using simulation. Am J Obstet Gynecol 2011;204(03):234.e1-234.e5

5 Gonik B, Zhang N, Grimm MJ. Prediction of brachial plexus stretching during shoulder dystocia using a computer simulation model. Am J Obstet Gynecol 2003;189(04):1168-1172 
6 Lerner HM. Shoulder dystocia: What is the legal standard of care? OBG Manag 2006;18:56-68

7 Gilbert WM, Nesbitt TS, Danielsen B. Associated factors in 1611 cases of brachial plexus injury. Obstet Gynecol 1999;93(04):536-540

8 Gherman RB, Ouzounian JG, Goodwin TM. Brachial plexus palsy: an in utero injury? Am J Obstet Gynecol 1999;180(05):1303-1307

9 Crofts JF, Ellis D, James M, Hunt LP, Fox R, Draycott TJ. Pattern and degree of forces applied during simulation of shoulder dystocia. Am J Obstet Gynecol 2007;197(02):156.e1-156.e6
10 Wilson L, Ash J, Crofts J, Sibanda T, Draycott T. Does training reduce the incidence of fetal injury in cases of shoulder dystocia? Simul Healthc 2006;1(03):185

11 Draycott TJ, Crofts JF, Ash JP, et al. Improving neonatal outcome through practical shoulder dystocia training. Obstet Gynecol 2008;112(01):14-20

12 Deering S, Poggi S, Macedonia C, Gherman R, Satin AJ. Improving resident competency in the management of shoulder dystocia with simulation training. Obstet Gynecol 2004;103(06):1224-1228 